\title{
Models for Wolf-Rayet and O star populations in starbursts: new developments and applications to large samples of Wolf-Rayet galaxies
}

\author{
Daniel Schaerer \\ Observatoire Midi-Pyrénées, \\ 14, Av. E. Belin, F-31400, Toulouse, France
}

\begin{abstract}
We summarise recent developments on synthesis models for massive star populations with a particular emphasis on Wolf-Rayet stars. Quantitative analyses of the stellar content of WR galaxies are reviewed. Comparing observations of WR galaxies from various samples with synthesis models we derive constraints on their burst properties. The observations indicate very short burst periods and are generally compatible with a Salpeter IMF and a large upper mass cut-off. The use of the $\mathrm{H} \beta$ equivalent width as an age indicator works well for WR galaxies. We briefly summarise comparisons of stellar populations in super star clusters which also provide useful contraints on evolutionary models for massive stars e.g., at very low metallicities inaccessible in the Local Group. In particular the observed WN and WC populations favour the high mass loss models of Meynet et al. (1994). Finally we review recent work on the origin of nebular He II emission. From the new catalogue of WR galaxies and high excitation HII regions of Schaerer et al. (1999) we find a close relation between the presence of nebular He II and WR stars. The analysis of individual WR galaxies including IZw 18 supports the suggestion of Schaerer (1996) that hot WR stars are responsible for the hard ionizing flux.
\end{abstract}

\section{Synthesis models for young starbursts}

There exist several independent synthesis models suited to the analysis of massive star populations in WR galaxies in particular ( $c f$. review of Leitherer, these Proceedings). A subset of models predict essentially the relative distributions of different populations (e.g., WN, WC, O stars), $\mathrm{SN}$ rates, etc. (e.g., Meynet 1995, Vanbeveren et al. 1997, 1998), whereas no (or very limited) direct predictions about the spectral properties or other observables are made. The models of Cerviño \& Mas-Hesse (1994), Leitherer \& Heckman (1995) or the updated/extended version (STARBURST99: Leitherer et al. 1999), Schaerer (1996) and Schaerer \& Vacca (1998), on the other hand predict numerous observational features including at least the hydrogen recombination lines and the strength of WR feature(s), essential to the interpretation of the massive star content in young starbursts.

For the following we shall rely on the recent synthesis models of Schaerer \& Vacca (1998, hereafter SV98) who predict the strongest WR lines in the UV and optical: He II $\lambda 1640, \mathrm{~N}$ III $\lambda 4640, \mathrm{C}$ III $\lambda 4650, \mathrm{He}$ II $\lambda 4686, \mathrm{He}$ II $+\mathrm{H} \beta \lambda 4861$, He II $\lambda 5412$, C III $\lambda 5696, \mathrm{C}$ IV $\lambda 5808, \mathrm{He}$ II $+\mathrm{H} \alpha \lambda 6560$. The contribution from WNL, WNE, WC4-9, and WO stars to these lines is taken into account in the 
models based on newly determined empirical line luminosities (see SV98). The He II $\lambda 4686$ emission from Of stars and Of/WN stars found in young clusters (e.g., R 136, NGC 3603, cf. Heap, Drissen, these Proceedings) is also considered (see SV98, Schaerer et al. 1999, hereafter SCK99). The SV98 models allow in particular direct comparisons with the He II $\lambda 4686$ line which is preferred over the use of the so-called broad 'WR bump', well known to consist in general of a blend of several stellar (WR) but also nebular lines rendering the interpretation of its strength difficult. The use of other independent WR lines (e.g., C III $\lambda 4650$, C IV $\lambda 5808$ ) now observed increasingly often ( $c f$. SCK99, compilation of Schaerer et al. 1999) furthermore allows one to distinguish between WN and WC subtypes.

An important 'feature' of these synthesis models is that they rely on stellar evolution tracks (Geneva models) which have extensively been compared with observations of both individual WR stars in the Galaxy and the LMC as well as WR and O star populations in the Local Group (Maeder \& Meynet 1994). These comparisons in particular favour the high mass-loss models (Meynet et al. 1994) generally used here. It is understood that although the adopted mass loss rates in the WR phases are likely too high compared to observations including the effects of clumping (e.g., Hillier, these Proceedings) this effect may be compensated by additional mixing processes leading to a similar prolongation of the WR phase (cf. Maeder, Meynet, these Proceedings).

\section{Analysis of WR and O-type stars in starbursts}

The latest catalogue of WR galaxies (Schaerer et al. 1999, hereafter SCP99) includes now nearly 140 objects (mostly giant HII regions, BCD, etc., but also some starburst nuclei, IRAS galaxies, etc.) However, so far there have been fairly few quantitative analyses of the WR and $\mathrm{O}$ star content and the properties of these regions. The aim of this Section is to summarise previous work and extend currently existing studies to a larger sample.

The bulk of data available in the literature on the WR signature in integrated spectra is summarised in Fig. 1 (left), showing the relative intensity of the WR bump (He II $\lambda 4686$ ) in the top (bottom) panel as a function of metallicity. In the early study of Arnault et al. (1989) the top figure was already interpreted as showing the following: (1) The spread in $\mathrm{WR} / \mathrm{H} \beta$ at a given $\mathrm{O} / \mathrm{H}$ is due to different ages; (2) There is an increase of the envelope of $\mathrm{WR} / \mathrm{H} \beta$ with $\mathrm{O} / \mathrm{H}$, which can be simply understood by the decrease of the mass limit for WR formation towards higher metallicity already shown by Maeder et al. (1980); and (3) Their first quantitative modeling also indicated that short star formation periods seemed to be required to achieve the large observed WR bump intensities.

Several subsequent studies have secured spectra with sufficient resolution and $\mathrm{S} / \mathrm{N}$ to allow a reliable measurement of the broad He II $\lambda 4686$ line in particular to circumvent the above mentioned difficulties associated with the WR bump. In passing we note from Fig. 1 (left, bottom) that He II $\lambda 4686 / \mathrm{H} \beta$ from these samples shows the same trends as discussed above. Kunth \& Sargent (1981), VC92 and Vacca (1994) put forward the determination of the number of $\mathrm{WN}$ and equivalent $\mathrm{O}$ stars as a convenient way to quantify the massive star 

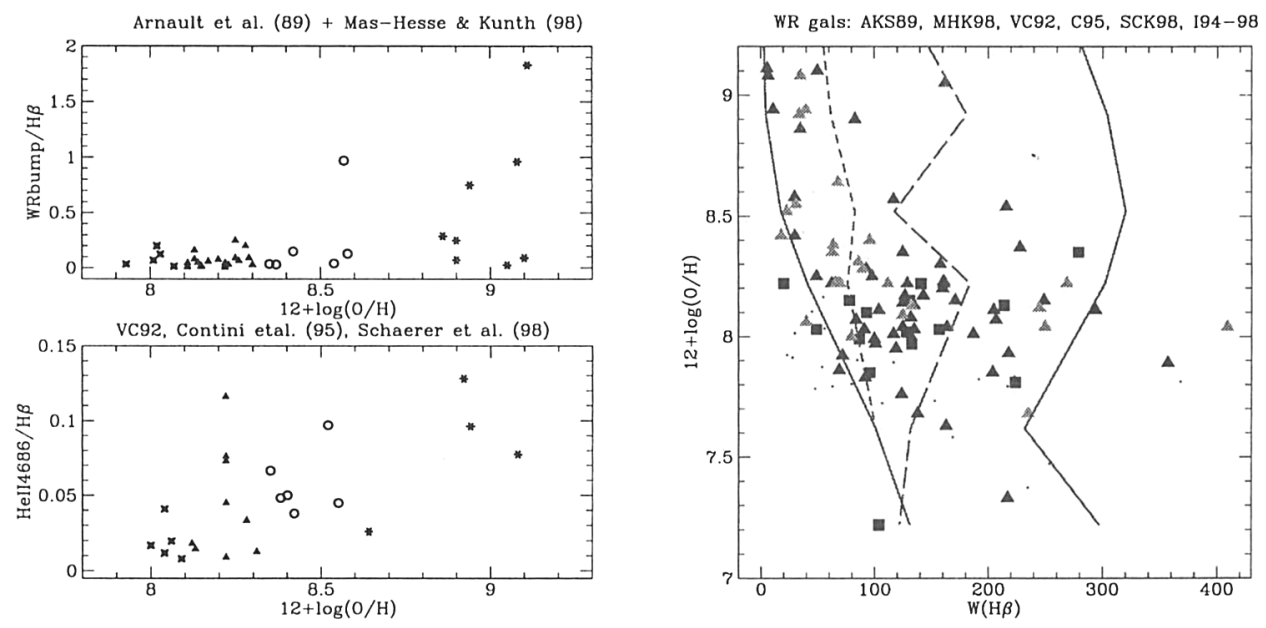

Figure 1. Left - Top: WR bump/ $\mathrm{H} \beta$ intensity as a function of $\mathrm{O} / \mathrm{H}$ for the objects from Arnault et al. (1989) and Mas-Hesse \& Kunth (1999). Bottom: Same for He II $\lambda 4686 / \mathrm{H} \beta$ from the samples of Vacca \& Conti (1992), Contini et al. (1995) and SCK99. Different symbols represent different metallicity 'bins' (see Fig. 2). Right: Position of WR galaxies in metallicity $(\mathrm{O} / \mathrm{H})$ vs. $\mathrm{H} \beta$ equivalent width (triangles, squares). Different greytones/colours indicate different samples. Solid lines denote the beginning/end of the WR-rich phase predicted by the SV98 models. Dashed lines indicate age steps of $1 \mathrm{Myr}$ during the WR phase.

populations. Although affected by several uncertainties and systematic effects ( $c f$. below) this simple method is still often used. Comparisons of the WN/O number ratios of VC92 with simple 'number' synthesis models clearly show that very short ( $\sim$ instantaneous compared to the lifetime of massive stars) bursts of star formation are required to reproduce the large $\mathrm{WN} / \mathrm{O}$ values (e.g., Maeder \& Conti 1994; Meynet 1995). The observed variation of WN/O with metallicity is also fairly well reproduced by these models.

The major drawback of quantitative comparisons of 'observed' WN/O number ratios with evolutionary models stems from the determination of the number of $\mathrm{O}$ stars (from an $\mathrm{H}$ recombination line), which is dependent on the IMF, star formation history, and age of the population ( $c f$. Vacca 1994, SV98). Indeed as shown by Schaerer (1996) the method of VC92 and Vacca (1994) leads to a systematic underestimate of the number of $\mathrm{O}$ stars. Comparisons of WR/O number ratios must therefore be taken with caution e.g., when used to constrain the IMF slope ( $c f$. Contini et al. 1995; Schaerer 1996).

It is encouraging that the disadvantage of the previous method can be easily circumvented without the need to invoke any additional major assumption. Evolutionary synthesis models (such as those discussed above) can directly predict the observable quantities (He II $\lambda 4686$ and $\mathrm{H} \beta$ line intensities) for given SFR, IMF and age - the assumption relating the ionizing photon flux to $\mathrm{H} \beta$ (e.g., case $\mathrm{B}$ recombination) remains as before. Comparisons with observations can 
thus directly be made in a plane of observables. Results from such studies are presented in the next section.

\subsection{Comparisons with recent synthesis models}

Uncertainties: Before we proceed it is useful to remember the most important potential observational and theoretical uncertainties involved in this process $(c f$. Conti, these Proceedings). The measurement of relative $\mathrm{WR} / \mathrm{H} \beta$ line intensities and equivalent widths can mostly be affected by three effects (see SCK99): (i) Not all ionizing photons are counted (e.g., small slit, leakage, etc.); (ii) Stars and gas suffer from a different extinction; and (iii) An underlying older population contributes additional continuum light. Some uncertainties due to the synthesis models (stellar tracks excluded) are (SCK99): (a) The use of the intrinsic WR line luminosity which shows e.g., a relatively large scatter for He II $\lambda 4686$ (SV98, Fig. 1); (b) Interpolation of tracks leading to uncertainties in the relative WC to WN populations; and (c) Assumptions on the calculation of $\mathrm{H}$ recombination lines.

On the $W(\mathrm{H} \beta)$ age indicator for WR galaxies: In Fig. 1 (right) we have plotted the position of all WR galaxies from the samples of Arnault et al. (1989), Mas-Hesse \& Kunth (1999), VC92, Contini et al. (1995), SCK99, and Izotov and collaborators (see references in Izotov \& Thuan 1998) in the $W(\mathrm{H} \beta)$ metallicity plane. Note that the bulk of these objects represents star forming regions (e.g., giant HII regions, etc.) in BCDs or spirals. If the $\mathrm{H} \beta$ equivalent width is taken as an age indicator, the temporal evolution proceeds to the left in this plot. Overplotted are the predictions from the SV98 models for $W(\mathrm{H} \beta)$ at the beginning and end of the WR phase for instantaneous burst models with a Salpeter IMF and $M_{\text {up }}=120 \mathrm{M}_{\odot}$. We note that essentially all WR galaxies lie within the predicted $W(\mathrm{H} \beta)$ range; in the most populated metallicity range $(12+\log (\mathrm{O} / \mathrm{H}) \simeq 7.8-8.5)$ the observations also fully populate this domain. Although it must be noted that each individual observation of $W(\mathrm{H} \beta)$ may be subject to the uncertainties mentioned above, we conclude from Fig. 1 (right) that on average the age and duration of the WR-rich phase predicted by the SV98 models for instantaneous bursts agree quite well with the observations. In this context it must, however, be noted that regions with very large $\mathrm{H} \beta$ equivalent widths such as predicted for populations of ages $\sim 0-1 \mathrm{Myr}$ are not observed. The origin of this well known discrepancy is not yet understood.

Observations of the WR bump: In Fig. 2 (left) we have plotted WR bump/H $\beta$ intensities from the samples of Arnault et al. (1989) and Mas-Hesse \& Kunth (1999). When two measurements are available we used more recent observations of Mas-Hesse \& Kunth; also the WR bump intensities corrected for the underlying population and differential extinction were used (see Mas-Hesse \& Kunth 1999). Note also that higher resolution spectra measuring He II $\lambda 4686$ are available for many of these objects (see below). Comparing these observations with the model predictions at the appropriate metallicity shows the following:

1) Most objects have WR bump intensities around or below the model prediction indicating that the observed WR and $\mathrm{O}$ star populations are reasonably well reproduced by the models calculated for instantaneous bursts. 

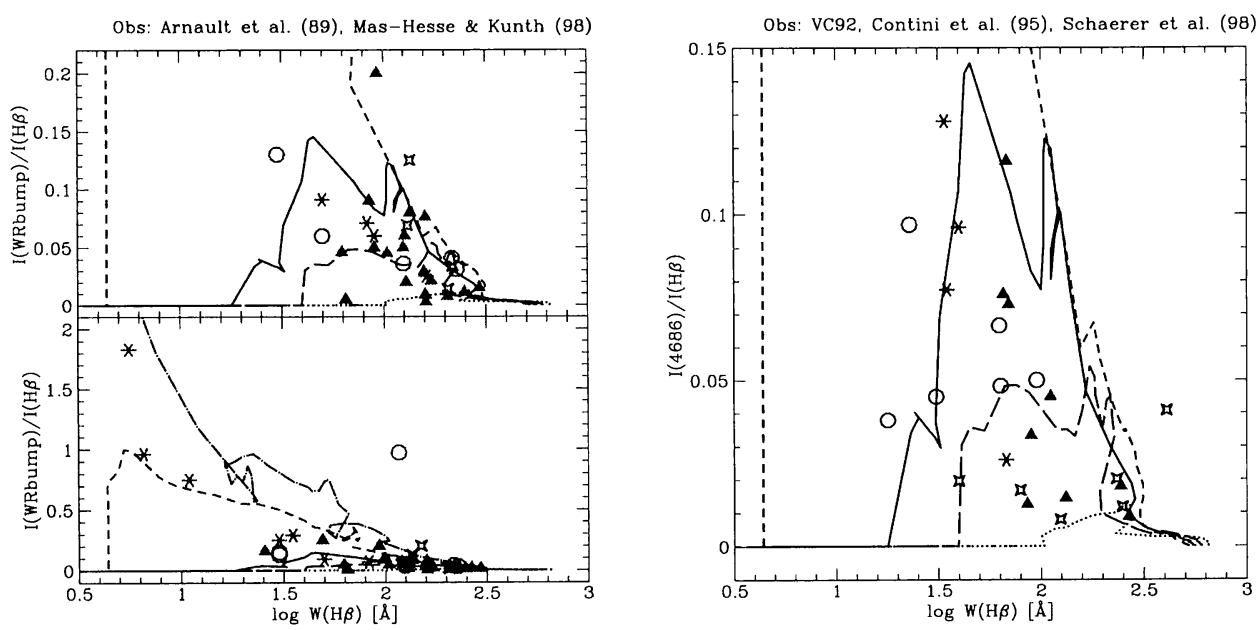

Figure 2. Left: Comparison of relative WR bump/H $\beta$ intensities from various samples (see text) with synthesis models of SV98 (instantaneous burst, Salpeter IMF). The upper panel shows a blow-up of the bottom. Right: Same as left for He II $\lambda 4686 / \mathrm{H} \beta$. Samples described in Sect. 3.2. In all Figures metallicities are indicated by different symbols: stars $(Z>0.01)$ to be compared with dashed and dott-dashed lines (models at $Z=0.02$ and 0.04 , resp.), circles $(0.005<Z \leq 0.01)$ to be compared with solid line $(Z=0.008$ models $)$, filled triangles $(0.003<Z \leq 0.05)$ vs. long-dashed $(Z=0.004$ models $)$, 4-star $(Z<0.003)$ vs. dotted $(Z=0.001$ models $)$.

2) The largest WR bump/ $\mathrm{H} \beta$ are found in objects with higher metallicity. This can be understood in the following way: The strong $\mathrm{WR} / \mathrm{H} \beta$ increase is mostly due to the decrease of $\mathrm{H} \beta$; the equivalent width of the WR features increase much more slowly ( $c f$. SV98). The rapid decrease of $\mathrm{H} \beta$ in turn is naturally predicted by evolutionary models at solar and higher metallicity, due to a cooler ZAMS and an extension of the MS to lower temperatures (see SV98). Such a metallicity effect on MS stellar evolution is also supported by studies of the nebular properties of Hil galaxies (e.g., García-Vargas et al. 1995; Stasińska \& Leitherer 1996). Other effects like dust could, however, also provoke such changes with metallicity as pointed out by T. Heckman (these Proceedings). Studies of larger samples of high metallicity regions and detailed multi-wavelength analyses would be of interest.

3) A few objects show WR bump intensities largely in excess of the model predictions $^{1}$. For some of them (e.g., NGC 1741) later measurements of He II $\lambda 4686$ (see below) show no discrepancy. Other data (e.g., from Rosa \& D'Odorico 1986) are quite uncertain. There remain, however, some objects with high quality measurements and an 'excessively' strong He II $\lambda 4686$, which might

${ }^{1}$ E.g., in Fig. 2 NGC 1741: $(\log W(\mathrm{H} \beta), \mathrm{WR} / \mathrm{H} \beta) \simeq(2 ., 0.2) ;$ Tol 89: $(2.1,0.13) ;$ Mrk 4038 \#3: $(2 ., 1)$.$) .$ 
be indicative of an unusual IMF (e.g., Tol 89, see SCK99 and below, also Huang et al. 1999).

\subsection{IMF and burst duration from individual Wolf-Rayet lines}

In Fig. 2 (right) we plot the majority of the observations where a measurement of He II $\lambda 4686$ is available. Using this line instead of the WR bump together with additional WR lines (see SCK99) should allow more accurate comparisons and provide e.g., even a reasonable handle on the IMF slope. A comparison of the observations of VC92 with the SV98 models has been presented by Schaerer (1996). New high-S/N spectra of NGC 5253, NGC 3049, NGC 3125, He2-10 and Tol 89 were analysed in detail by Schaerer et al. (1997) and SCK99. Here we briefly summarise their main results (see also Contini et al. these Proceedings).

Inspection of Fig. 2 shows that the vast majority of the observed He II $\lambda 4686$ intensities are well reproduced by the models at the appropriate metallicity assuming an instantaneous burst and a Salpeter IMF. However, some objects show large He II $\lambda 4686 / \mathrm{H} \beta$. Is this convincing evidence calling e.g., for a flatter $I M F$ ? We think the answer is no. As pointed out by SCK99 it is useful to compare also the equivalent widths of the WR feature(s) since these are affected differently by the uncertainties listed above. Indeed in some objects there is evidence showing a different spatial distribution of the gas and stars (SCK99): in this case the use of $\mathrm{WR} / \mathrm{H} \beta$ line intensities may not be reliable and it is preferable to use quantities related only to the stellar content (i.e., $W(\mathrm{WR})$ ). Furthermore the detection of several 'independent' WR lines (He II, C IV) increases the number of constraints on the models.

The detailed comparisons of SCK99 taking these effects into account yield the following: (1) Essentially instantaneous bursts $(\Delta t \lesssim 2-4 \mathrm{Myr})$ are required to reproduce the observed WR line intensities; (2) The majority of the observations can be reproduced with a Salpeter IMF; no clear case requiring a flatter IMF is found; (3) The IMF in these regions must be populated up to large masses. For the case of $\mathrm{IZw} 18$, at $Z \simeq 0.02 \mathrm{Z}_{\odot}, M_{\text {up }}$ extending to $120-150 \mathrm{M}_{\odot}$ is found (de Mello et al. 1998); and (4) The relative populations of WN and WC stars detected clearly favour the high mass evolutionary models (SCK99).

We note that our results on the IMF slope and the upper mass cut-off are in agreement with several independent studies, e.g., stellar counts (review by Massey 1998), UV line profile modeling of starbursts (references in Leitherer 1998), photoionization models for Hir galaxies (e.g., García-Vargas et al. 1995, Stasińska \& Leitherer 1996), etc. Interestingly, however, there are several studies indicating the possibility of a lower value for $M_{\mathrm{up}}$ at metallicities above solar (e.g., ULIRG: see review of Leitherer 1998; H II regions: Bresolin et al. 1999). A search for WR stars in metal rich regions should allow one to put direct constraints on the upper end of the IMF in such environments.

\section{The origin of nebular He II $\lambda 4686$ emission}

The presence of nebular He II $\lambda 4686$ emission indicative of high excitation in extra-galactic HII regions and WR galaxies has attracted new attention in recent years. Here we shall briefly summarise the current status and recent work aiming at understanding the nature of this emission (see also Schaerer 1996, 1998). 
These studies have a direct bearing on our understanding of the properties of WR stars, the structure and physics of HII regions, and our knowledge of the ionizing fluxes of starbursts. Some of these aspects are also discussed by Crowther; Galarza et al.; Peña et al.; Stasińska; and others in these Proceedings.

\subsection{Empirical facts}

In the Local Group eight nebulae exhibit nebular He II $\lambda 4686$ emission indicative of unusually high excitation. All except one Galactic object are located in low metallicity environments (IC 1613, SMC, LMC). Except for two cases the nebulae are associated with early WN and WO stars. Approximately 60 objects (mostly giant HII regions) outside the Local Group with nebular He II $\lambda 4686$ are now known (see compilation of Schaerer et al. 1999, SCP99). Most of them have low metallicities, and typically $I(4686) / I(\mathrm{H} \beta) \simeq 1-5 \%$. Many objects where originally nebular He II $\lambda 4686$ was reported, have revealed the presence of broad He II $\lambda 4686$ in spectra with better $\mathrm{S} / \mathrm{N}$ and resolution, and are therefore classified as WR galaxies. Many WR galaxies also exhibit simultaneously nebular He II $\lambda 4686$ emission. These observations strongly suggest that the phenomenon of nebular He II emission is related to the presence of WR stars.

\subsection{Quantitative models}

In this context Schaerer (1996) presented the first models aiming at a quantitative explanation of nebular He II emission in WR galaxies and related objects. Indeed, it is found that synthesis models using appropriate model atmospheres for WR and O stars (see also SV98) are able to produce nebular He II with intensities in the range of the observations during a relatively short phase where hot WN and WC/WO stars are present. This is in agreement with the presence of nebular He II $\lambda 4686$ in several WR galaxies (see Schaerer 1996; Mas-Hesse \& Kunth 1999). It is also supported by the detection of WR stars in I Zw 18 (Legrand et al. 1997; Izotov et al. 1997), the galaxy with the lowest metal content known: using appropriate stellar tracks one is able to reasonably reproduce the WR content and nebular He II $\lambda 4686$ emission in this galaxy (de Mello et al. 1998; Stasińska, these Proceedings). At intermediate to high metallicities He II $\lambda 4686$ is predicted to be largely dominated by stellar emission from WR stars (Schaerer 1996, SV98). In addition, compared to the atmosphere models used in SV98 the blanketing effects discussed by Crowther (these Proceedings) are also likely to progressively reduce the high energy photon flux of WR stars with increasing metallicity. Under reasonable assumptions and using evolutionary synthesis models one can also rule out several other potential sources of He II $\lambda 4686$ emission ( $c f$. Garnett et al. 1991): Of stars, O stars close to the Eddington limit, and X-ray binaries. We conclude that synthesis models including appropriate model atmospheres are able to reproduce the observed nebular He II $\lambda 4686$ emission when WR stars are present.

Independent information supporting WR stars as the origin of He II ionizing photons comes from (admittedly scarce) spatial information: When observed, the spatial distribution of nebular He II $\lambda 4686$ follows that of the WR features (Legrand et al., Izotov et al., Maiz-Apellaniz et al., de Mello et al.). Statistically one can also make the following arguments (see samples in SCP99): (1) In the sample of Izotov and collaborators 40 objects show nebular He II; $75 \%$ of them 
show also WR features (half of them WN and WC). Conversely, from the 18 objects without nebular He II only $28 \%$ harbour WR stars; and (2) In the total sample of SCP99 aiming at a complete list of He II $\lambda 4686$ detections from the literature, 54 objects exhibit both broad (WR) and nebular He II $\lambda 4686$, whereas only 19 HII regions show purely nebular He II. Clearly the vast majority of objects showing nebular He II harbour WR stars. This corroborates the results discussed above.

Analyses of the Local Group objects allowing a detailed investigation of the stellar content and the physical processes in the nebula would be rewarding to test these results. Further studies of the HII regions with purely nebular He II need also to be undertaken.

\section{Conclusions}

We have summarised recent developments on synthesis models for massive star populations with a particular emphasis on WR stars. The SV98 models rely in particular on up-to-date atmospheres and stellar tracks which have extensively been compared to observations in the Galaxy and the Local Group. Comparisons of observations of WR galaxies with these and other synthesis models yield the following main results: (1) The predicted age and duration of the WR-rich phase agree well with the observations; (2) Observations of high WR bump/H $\beta$ intensities in high metallicity objects seem to be due to a low $\mathrm{H} \beta$ flux. This can be explained by metallicity effects on the stellar tracks although other explanations are also possible; (3) The observed WR and $\mathrm{O}$ star populations in all objects indicate nearly instantaneous bursts; (4) The vast majority of the objects can be reproduced with a Salpeter slope for the IMF. The upper mass cut-off is large in these galaxies such as to produce enough WR stars; (5) The quantitative analysis of both WN and WC populations in WR galaxies supports high mass-loss evolutionary tracks. Other mechanisms (e.g., rotational mixing) may have similar effects; and (6) As shown by the example of IZw 18 (de Mello et al. 1998), the analysis of stellar populations in super star clusters may provide useful constraints on the evolution of massive stars, especially at low metallicities inaccessible in the Local Group.

The origin of nebular He II emission requiring copious ionizing photons with energies above $54 \mathrm{eV}$ has been puzzling. We find that the observations in the Local Group and more distant objects suggest a close relationship between the presence of nebular He II $\lambda 4686$ emission and WR stars. Quantitatively the observed nebular He II can be explained by synthesis (and photoionization) models due to the presence of hot WN and/or WC/WO stars (Schaerer 1996; de Mello et al., Stasińska, these Proceedings).

The work summarized here should improve our knowledge not only on the evolution and atmospheres of massive stars, but also on starbursts and their properties.

Acknowledgments. DS acknowledges a grant from the Swiss National Foundation of Scientific Research and financial support from the IAU and the GdR Galaxies. 


\section{References}

Arnault, P., Kunth, D., Schild, H. 1989, A\&A 224, 73

Bresolin, F., Kennicutt, R.C., Garnett, D.R. 1999, ApJ 510, 104

Cerviño, M., Mas-Hesse, J.M. 1994, A\&A 284, 749

Contini, T., Davoust, E., Considère, S. 1995, A\&A 303, 440

de Mello, D.F., Schaerer, D., Heldman, J., Leitherer, C. 1998, ApJ 507, 199

García-Vargas, M.L., Bressan, A., Díaz, A.I. 1995, A\&AS 112, 13

Garnett, D.R., Kennicutt, R.C., Chu, Y.H., Skillman, E.D. 1991, ApJ 373, 458

Huang, J.H., Gu, Q.S., Ji, L., Li, W.D., Wei, J.Y., Zheng, W. 1999, ApJ 513, 215

Izotov, Y.I., Thuan, T.X. 1998, ApJ 497, 227

Izotov, Y.I., et al. 1997, ApJ 487, L37

Kunth, D., Sargent, W.L.W. 1981, A\&A 101, L5

Legrand, F., et al. 1997, A\&A 326, L17

Leitherer, C. 1998, in: G. Gilmore \& D. Howell (eds.), The Stellar Initial Mass Function, ASP-CS 142, 61

Leitherer, C., Heckman, T.M. 1995, ApJS 96, 9

Leitherer, C., et al. 1999, ApJS submitted

Maeder, A., Conti, P.S. 1994, ARA\&A 32, 227

Maeder, A., Lequeux, J., Azzopardi, M. 1980, A\&A 90, L17

Maeder, A., Meynet, G. 1994, A\&A 287, 803

Maiz-Apellaniz, J., et al. 1998, A\&A 329, 409

Mas-Hesse, J.M., Kunth, D. 1999, A\&A in press (astro-ph/9812072)

Massey, P. 1998, in: G. Gilmore \& D. Howell (eds.), The Stellar Initial Mass Function, ASP-CS 142,17

Meynet, G. 1995, A\&A 298, 767

Meynet, G., Maeder, A., Schaller, G., Schaerer, D., Charbonnel, C. 1994, A\&AS 103, 97

Schaerer, D. 1996, ApJ 467, L17

Schaerer, D. 1998, in: J. Andersen (ed.), Highlights of Astronomy, Vol. 11 (Dordrecht: Kluwer), p. 134

Schaerer, D., Contini, T., Kunth D. 1999, A\&A 341, 399 (SCK99)

Schaerer, D., Contini, T., Kunth D., Meynet G. 1997, ApJ 481, L75

Schaerer, D., Contini, T., Pindao M. 1999, A\&AS 136, 35 (SCP99)

Schaerer, D., Vacca, W.D. 1998, ApJ 497, 618 (SV98)

Stasińska, G., Leitherer, C. 1996, ApJS 107, 661

Vacca, W.D. 1994, ApJ 421, 140

Vacca, W.D., Conti, P.S. 1992, ApJ 401, 543 (VC92)

Vanbeveren, D., Van Bever, J., De Donder, E. 1997, A\&A 317, 487

Vanbeveren, D., De Donder, E., Van Bever, J., Van Rensbergen, W., de Loore, C. 1998, New Astronomy 3, 443

\section{Discussion}

Vanbeveren: Most of the WR stars in the SMC are binaries. Before drawing conclusions as far as the WR content in $\mathrm{IZw} 18$ is concerned, which are based on single-star evolution, wouldn't it be advisable to investigate the effect of binaries first? 
Schaerer: It is certainly interesting to investigate the formation of WR stars through RLOF. The most fundamental test cases to do this are obviously stellar clusters, which should ideally be used as the first step. For IZw 18 I do not think that RLOF in high-mass binaries plays an important role. See answer to Kunth, below.

Walborn: (1) WN stars have C-lines (saturated C IV 1550 wind-profiles in WNLs, but also C IV 5800 emission lines), so if you look hard enough you will see them. (2) Few or no WCs occur in local giant $\mathrm{H}$ II regions, indicating they correspond predominantly to later evolutionary stages. I suggest that UV-bright but $\mathrm{H} \alpha$-weak starburst-knots will show more WC contributions than $\mathrm{H} \alpha$-bright regions. (3) Eventually, when the observational and theoretical uncertainties warrant, instantaneous starburst models will have to incorporate the two- or three-stage concentric bursts shown by all nearby, resolved starburst regions. For example, again, the central Sco OB1 association of $\mathrm{N} 11$ has a WC star, while the surrounding Carina phase has not yet produced a WN!

Lehnert: Can you comment on the role of dust in perhaps softening the ionizing spectrum in starburst galaxies?

Schaerer: To the best of my knowlegde very little work has been done in this respect. See also answer to Heckman's comment, below.

Kunth: Although your single-star population model satisfactorily accounts for the WC/WN population of low-metallicity galaxies (such as IZw 18), can you completely rule out the binary-channel solution?

Schaerer: The simple answer is obviously: no. However, e.g., using $W(\mathrm{H} \beta)$ as an age estimate (taking the case of the NW region of $\mathrm{IZw} 18$ of the extended $\mathrm{H} \beta$ emission not included in many slit-spectra), one obtains a fairly young age for this region in IZw 18 $(\sim 2-4 \mathrm{Myr})$. According to the existing binary star models, this age is too young for the formation of WR stars through RLOF.

Langer: Can you, with your WR galaxy models, constrain the metallicity $(Z)$ dependence of the mass loss rates of WR stars? E.g., you showed observational evidence for the $Z$-dependence of the strengths of the WR emission-line features in WR galaxy spectra. Could part of this feature be due to $Z$-dependent WR mass loss, in the sense that if WR stars at lower $Z$ have weaker winds, then they should also have weaker emission features?

Schaerer: The average line luminosity of WN stars in He II4686 does not show a significant difference between the Galaxy (objects with known distance), the LMC and SMC (see Schaerer \& Vacca 1998 for Galaxy, LMC). Therefore, the main reason for the decrease of the observed He II strength is due to the diminished WR population at low $Z$. Given the various uncertainties (observations and models) I do not think that it is currently possible to constrain $\dot{M}(Z)$ of WR stars for which there is presently no indication in analyses of individual stars.

Maeder: In the case of $\mathrm{IZw} 18$, it seems to me that in order to account for the estimated $\mathrm{WR} / \mathrm{O}$ ratio of 0.02 , we need some effects of rotation or binarity in addition to our usual predictions, which gives something smaller than the above value. At very low $Z$, the balance between the various scenarios for forming WR stars is not necessarily the same as at $Z_{\odot}($ e.g., Maeder 1981).

Schaerer: For I Zw 18 one cannot use the WR/O ratio determined from the observations of Legrand et al. (1997) and/or Izotov et al. (1997), since the slit includes only a small fraction of the $\mathrm{H} \beta$ flux. This is easily seen in the HST images (see De Mello et al. 1998). Therefore, a comparison with quantities relating only to stars (not stars/gas) should only be used. In this case we showed that the equivalent widths of the WR lines are quite well reproduced with the single-star models discussed in De Mello et al. (1998). Although there is therefore no real need for other effects, this can, of course, not been excluded. 
Heckman: The work of Daniele Calzetti \& colleagues shows that the nebular emission in starbursts suffers significantly more extinction due to dust than the stellar continuum. Thus, the $\mathrm{H} \beta$ equivalent width will decrease as the dust content increases. The dust content is a strong function of metallicity. Thus, you should be very cautious about using the $\mathrm{H} \beta$ equivalent width to constrain the models or make inferences, especially in metal-rich (dusty) starbursts. In particular, the trend for the $\mathrm{H} \beta$ equivalent width to decrease with increasing metallicity may be due to just this effect. This could also affect the ratio of $\mathrm{H} \beta$ and WR bumps, again as a function of metallicity.

Schaerer: This is certainly a well-taken point, which should be investigated in more detail. E.g., Mas-Hesse \& Kunth (98) have partly examinated the importance of these effects on WR/H $\beta$. Certainly modelling in particular ISO observations of $\mathrm{H}$ II regions and starbursts together with other long-wavelength observations would be extremely interesting.

Schaerer: On the comment/question of Massey: The contribution of WN stars to C IV 5808 is taken into account in the Schaerer \& Vacca models. This is done using the empirical line fluxes newly determined for all the most important WR lines for WNE, WNL and WC/WO stars of all subtypes (see their Tables 1 and 2).

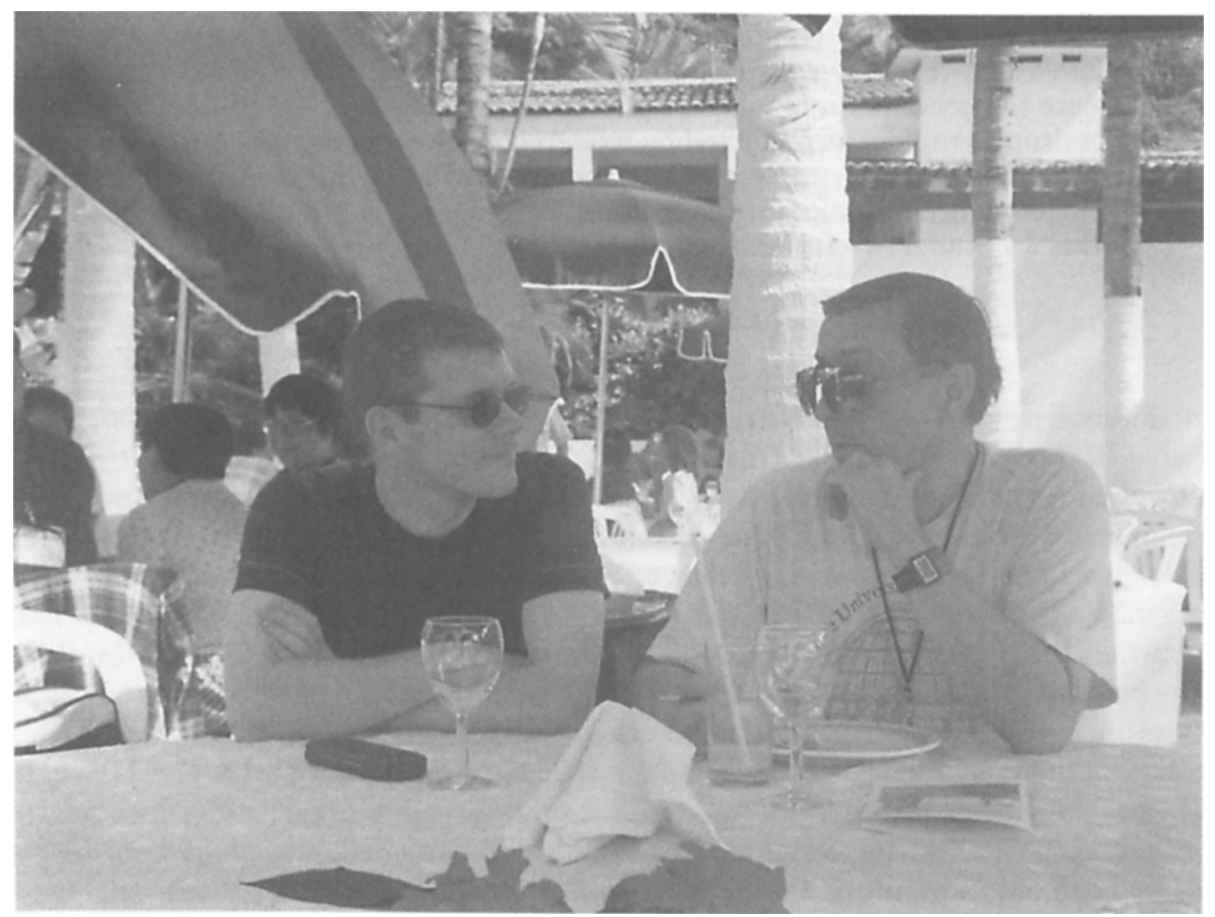

